The AstronOMical Journal, 116:2513-2519, 1998 November

(C) 1998. The American Astronomical Society. All rights reserved. Printed in U.S.A.

\title{
A DEEP MULTICOLOR SURVEY. V. THE M DWARF LUMINOSITY FUNCTION
}

\author{
Paul Martini and Patrick S. Osmer \\ Department of Astronomy, Ohio State University, 174 West 18th Avenue, Columbus, OH 43210; martini@astronomy.ohio-state.edu, \\ posmer@astronomy.ohio-state.edu \\ Received 1998 May 28; revised 1998 July 28
}

\begin{abstract}
We present a study of $\mathbf{M}$ dwarfs discovered in a large-area, multicolor survey. We employ a combination of morphological and color criteria to select $\mathbf{M}$ dwarfs to a limiting magnitude in $V$ of 22 , the deepest such ground-based survey for $\mathbf{M}$ dwarfs to date. We solve for the vertical disk stellar density law and use the resulting parameters to derive the $M$ dwarf luminosity and mass functions from this sample. We find that the stellar luminosity function peaks at $M_{V} \sim 12$ and declines thereafter. Our derived mass function for stars with $M<0.6 M_{\odot}$ is inconsistent with a Salpeter function at the $3 \sigma$ level; instead, we find that the mass function is relatively flat for $0.6 M_{\odot}>M>0.1 M_{\odot}$.

Key words: stars: late-type - stars: low-mass, brown dwarfs -

stars: luminosity function, mass function
\end{abstract}

\section{INTRODUCTION}

The study of $\mathbf{M}$ dwarfs in the disk of our Galaxy has applications to many areas of current research, including the determination of the mass of the disk, its star formation history, and the nature of the sources of observed microlensing events (e.g., Bahcall et al. 1994). The shape of the stellar luminosity function has been measured using a variety of techniques, including large-area, bright, groundbased surveys (e.g., Stobie, Ishida, \& Peacock 1989; Reid, Hawley, \& Gizis 1995; Reid \& Gizis 1997), deeper groundbased surveys with complementary spectroscopy (Reid et al. 1996), and space-based surveys performed with the Hubble Space Telescope (HST) (Gould, Bahcall, \& Flynn 1996, 1997). These have all shown that the shape of the stellar luminosity function peaks at $M_{V} \sim 12$ and declines thereafter to at least $M_{V} \sim 15$.

Each of these techniques has particular strengths. Largearea, ground-based surveys to relatively bright magnitude limits are particularly suited to determining the bright end of the $\mathrm{M}$ dwarf luminosity function in the solar neighborhood, where the effects of the space density gradient in the M dwarf population can be neglected. Deeper, groundbased surveys may be readily used to obtain a large sample of faint $\mathbf{M}$ dwarfs. These deeper surveys are generally limited to much smaller areas and can suffer from contamination from faint red galaxies. Deep, ground-based $\mathbf{M}$ dwarf counts therefore require spectroscopy to eliminate this contamination and to accurately measure the stellar luminosity function. Finally, star counts with $H S T$ can reach to the faintest limiting magnitudes by distinguishing stars from galaxies with the excellent angular resolution of $H S T$. Deep, space-based surveys preferentially sample $M$ dwarfs higher above the plane than ground-based, larger area surveys to brighter limiting magnitudes. They therefore probe both the shape of the stellar luminosity function and the space density of the M dwarf population, though they are not as sensitive to the brighter M dwarfs, as they probe smaller areas. These approaches therefore preferentially sample different regions of the phase space needed to determine both the $\mathrm{M}$ dwarf luminosity function and the space density of this population as a function of height above the plane and distance from the Galactic center.

In the present work, we derive the shape of the vertical stellar density distribution of $\mathrm{M}$ dwarfs and use this to compute their luminosity and mass functions using the deep multicolor survey (DMS) of Hall et al. (1996a). This survey has already been successfully used to study quasars (Hall et al. 1996b; Kennefick et al. 1997) and the luminosity function of galaxies (Liu et al. 1998). In this contribution we search the DMS stellar catalog (Osmer et al. 1998) for M dwarfs down to faint limiting magnitudes. However, in contrast to previous surveys, we primarily employ extensive multicolor information, rather than spectroscopy, to separate $\mathbf{M}$ dwarfs from faint red galaxies. We do, however, make use of the spectroscopic identifications obtained of selected $\mathbf{M}$ dwarfs as part of the search for quasars as a check of the efficiency of our multicolor selection criteria. Our final catalog of $M$ dwarfs extends to $V=22$ and contains a total of $1575 \mathrm{M}$ dwarfs, including 499 in bins over the range $12 \leq M_{V} \leq 15$.

\section{OBSERVATIONS AND SAMPLE SELECTION}

The multicolor survey fields obtained by Hall et al. (1996a) image six different high Galactic latitude $\left(|b|>35^{\circ}\right)$ fields in six filters and subtend a total of $0.83 \mathrm{deg}^{2}$. These six filters are Johnson $U B V$ and three special red filters, $R^{\prime}, I_{75}$, and $I_{86}$. The $R^{\prime}$ filter is somewhat narrower than a Harris $R$ or Cousins $R$ and has less of a red tail. The $I_{75}$ and $I_{86}$ filters are narrowband $I$ filters centered at 7500 and $8600 \AA$, respectively. Hall et al. (1996a) used FOCAS to catalog all of the stellar objects in these fields in order to search for quasars (Hall et al. 1996b; Kennefick et al. 1997). An object was declared stellar provided that it was classified as a star or fuzzy star in more than one-half of the filters in which it was detected, and each object was required to be detected in at least three filters for inclusion in the final stellar catalog. We note that this morphological criterion is extremely lenient because the goal was to not exclude active galactic nuclei, which may be slightly resolved; we do not expect it to have missed any stellar objects. The resulting catalog has $5 \sigma$ limiting magnitudes between 22.1 and 23.8 for these six filters. We refer the reader to Hall et al. (1996a) for a more detailed explanation of the filters, catalog construction, and limiting magnitudes summarized here. We summarize the field size and Galactic coordinates for each field in Table 1.

Reid et al. (1996), using the Low Resolution Imaging 
TABLE 1

Survey Field Characteristics

\begin{tabular}{crrr}
\hline \hline Field & $l$ & \multicolumn{1}{c}{$b$} & \multicolumn{1}{c}{$A$} \\
\hline $01 \ldots \ldots \ldots \ldots \ldots \ldots$ & 129 & -63 & 517.3 \\
$10 \ldots \ldots \ldots \ldots \ldots$ & 248 & 47 & 286.0 \\
$14 \ldots \ldots \ldots \ldots \ldots$ & 337 & 57 & 561.3 \\
$17 \ldots \ldots \ldots \ldots \ldots$ & 77 & 35 & 551.9 \\
$21 \ldots \ldots \ldots \ldots \ldots$ & 52 & -39 & 537.0 \\
$22 \ldots \ldots \ldots \ldots \ldots$ & 68 & -51 & 536.6 \\
\hline
\end{tabular}

Notes.-A summary of the six survey fields of Hall et al. 1996a. Col. (1) shows the field identification, which corresponds to the right ascension of the field in hours. Cols. (2) and (3) are the Galactic coordinates in degrees, and col. (4) lists the angular size of each field in square arcminutes.

Spectrometer on the Keck Telescope, find $R=22.5$ to be the limit for star-galaxy separation in their good-seeing $(0.5)$, ground-based data with a $10 \%$ contamination due to faint galaxies. They find the galaxy contamination to be $30 \%$ by $R=24.5$. The data discussed here had typical seeing between $1^{\prime \prime}$ and $2^{\prime \prime}$ FWHM, and we therefore use a combination of morphological and color criteria in order to extend the limiting magnitude of accurate star-galaxy separation as faint as possible. However, the three reddest filters used in this investigation are nonstandard, and therefore measurements of $\mathbf{M}$ dwarf colors with these filters do not exist in the literature. Fortunately, the $R^{\prime}$ filter has nearly the same effective wavelength as the KPNO Harris $R$ (which has the same transmission profile as the Cousins $R$, hereafter $R_{C}$ ), though slightly narrower and with less of a red tail. The $R^{\prime}$ magnitudes quoted by Hall et al. (1996a) are therefore equivalent to $R_{\mathrm{C}}$ except perhaps for the reddest objects, for which the $V-R^{\prime}$ color may be slightly bluer than $V-R_{\mathrm{C}}$ because of the weaker red tail of the $R^{\prime}$ filter.

To measure the magnitude of this potential color term, we used the STSDAS SYNPHOT package to convolve a sample of late-type stars from the Bruzual-Persson-GunnStryker Spectrophotometry Atlas ${ }^{1}$ with the $V, R_{\mathrm{C}}$, and $R^{\prime}$ filters. We found that the color term would be no greater than 0.02 mag in $V-R_{\mathrm{C}}$ for the reddest $\mathrm{M}$ dwarfs. As the standard stars used in the photometric calibration included stars as red as $V-R_{\mathrm{C}} \sim 1.3$ (P. B. Hall 1998, private communication), while the $\mathbf{M}$ dwarfs studied here have $0.9<V-R<1.6$, this is not surprising. It is also interesting to note the direction of this effect. The transformation from $R^{\prime}$ to $R_{\mathrm{C}}$ makes red objects brighter in the $R_{\mathrm{C}}$ filter. This corresponds to redder $V-R_{\mathrm{C}}$ and, hence, later spectral type and larger $M_{V}$. Thus any color term for the reddest objects would make them intrinsically fainter and therefore closer. Our assumption that $R^{\prime}$ is equivalent to $R_{\mathrm{C}}$ may therefore slightly increase the space density of the faintest $M$ dwarfs.

The transmission profiles and narrow widths of the $I_{75}$ and $I_{86}$ filters are significantly different from Cousins $I$. This region of the spectral energy distribution of $M$ stars is also quite strongly affected by the presence of molecular absorption. The combination of these two factors introduces significant uncertainties into a transformation of $I_{75}$ and $I_{86}$ to Cousins $I$, and we therefore did not perform this

\footnotetext{
${ }^{1}$ See http://www.stsci.edu/ftp/instrument_news/Observatory/cdbs/ astronomical_catalogs.html.
}

transformation; rather, this drove us to use the $V-R_{\mathrm{C}}$ color to estimate absolute magnitudes.

Ground-based surveys have been able to separate stars from galaxies to $R \sim 19$ without spectroscopic follow-up (Kirkpatrick et al. 1994). Koo, Kron, \& Cudworth (1986) used multicolor techniques to separate red stars from quasars to $V \sim 23$. To increase the limiting magnitude to which we could successfully discriminate $\mathbf{M}$ dwarfs from small red galaxies, we employed the five colors of the Hall et al. (1996a) data set to remove nonstellar objects. These filters effectively provide complete wavelength coverage from the atmospheric cutoff to $9000 \AA$. We expect stars and galaxies to separate in this five-dimensional color space. As we are searching for very red objects, however, requiring a detection in $U$ or even $B$ will significantly decrease the total number of objects in the sample extracted from the catalog. We therefore developed the following set of color and detection criteria for extracting a catalog of potential $\mathrm{M}$ dwarfs.

Our first cut was to require detection in $V, R_{\mathrm{C}}$, the two $I$ filters, and a $V$ magnitude less than 23.5, the $5 \sigma$ limiting magnitude in $V$ (though we refine this limit below). The purpose of this cut was to trim the size of the stellar catalog to objects with good signal-to-noise ratio photometry in the red filters. We then employed a second, much more discriminating cut to take advantage of our five-color information. We used the $\mathbf{M}$ dwarf color information tabulated by Leggett (1992) to set the following blue limits for a given star to be included in the $\mathbf{M}$ dwarf catalog, requiring that the star be within $3 \sigma$ of an M0 dwarf (or redder) in each color:

$$
\begin{gathered}
U-B+\left(3 \sigma_{U-B}\right)>1.15, \\
B-V+\left(3 \sigma_{B-V}\right)>1.35, \\
V-R_{\mathrm{C}}+\left(3 \sigma_{V-R_{\mathrm{C}}}\right)>0.90 .
\end{gathered}
$$

As stated above, we did not require that a given star be detected in the $U$ and $B$ filters. If the $1 \sigma$ photometric uncertainty for $U-B$ and/or $B-V$ color was greater than 0.333 , corresponding to a nondetection at the $3 \sigma$ level, we did not include this color criterion. Though $U$ and $B$ data were not available for every star in the sample, these filters were nevertheless useful for removing anomalously blue objects from our $\mathrm{M}$ dwarf sample. A blue $U-B$ color, for example, caused us to reject 290 stellar objects that we would have otherwise included in this sample, and a combination of blue $U-B$ and $B-V$ or $B-V$ alone caused us to reject an additional 120 stellar objects. We did not employ color selection criteria based upon the two $I$ filters. A quick visual inspection of color-color diagrams including these two bands (see Fig. 1), however, does not reveal a significant population of blue $\left(R_{\mathrm{C}}-I<0\right)$ outliers.

As an independent check of the efficiency of our color selection technique, we searched our final catalog for the $\mathbf{M}$ dwarfs "serendipitously" discovered in the course of the spectroscopic follow-up to search for faint quasars. These spectra, and the entire stellar catalog, are described by Osmer et al. (1998) and include 45 definite $M$ dwarfs with $V<22$. Our final $\mathrm{M}$ dwarf catalog includes 37 of these objects, or $82 \%$. The eight stars excluded by our search criteria were all eliminated because of their anomalous $U$ and $B$ brightness, suggesting they may have faint blue companions. As the presence of an ultraviolet excess is one of the selection criteria for quasars, it is not surprising that some of the M dwarfs observed spectroscopically are associ- 

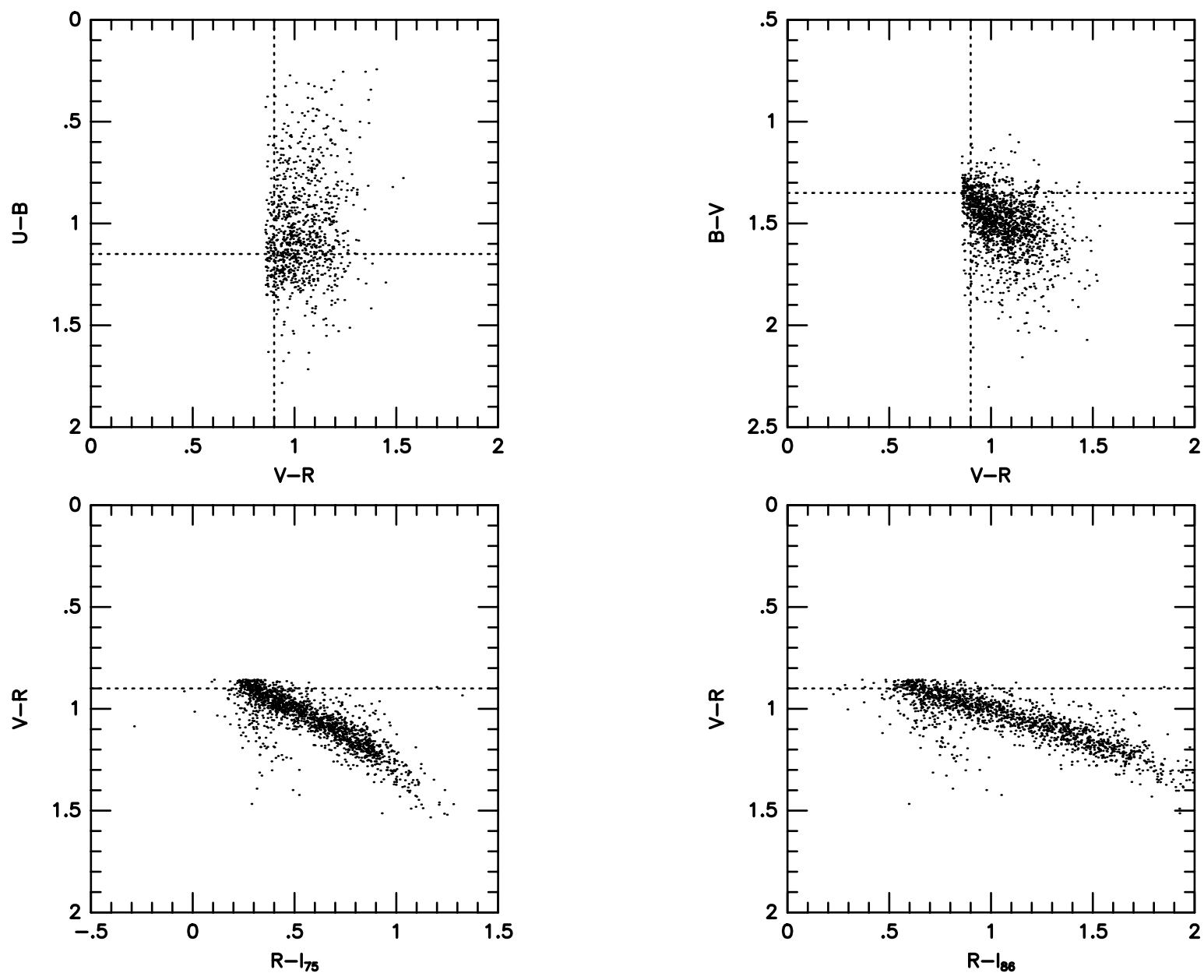

FIG. 1. Color-color diagrams of the $\mathrm{M}$ dwarf sample down to a limiting $V$ magnitude of 22. The top left panel shows $U-B$ vs. $V-R$, the top right panel shows $B-V$ vs. $V-R$, and the two lower panels show $V-R$ vs. $R-I_{75}$ and $R-I_{86}$, respectively. Photometric errors are generally 0.02 , increasing to 0.05 for objects near the magnitude limit. The dashed lines correspond to the color cutoffs for $\mathbf{M}$ dwarfs described in the text, with the $M$ dwarfs falling to the lower right in each plot. Stars outside of this range are consistent with these colors within $3 \sigma$.

ated with excess UV emission. In fact, the sample of $\mathbf{M}$ dwarfs with spectroscopic confirmation is biased toward stars with unusual UV colors, and this sample cannot be used to characterize the true efficiency of the color selection criteria for M dwarfs. Rather, the $82 \%$ completeness quoted above can only be considered a strong lower limit to the true completeness of this color-selected $\mathbf{M}$ dwarf sample.

\section{COLOR-MAGNITUDE RELATION}

We have employed the $V-R_{\mathrm{C}}$ color to estimate $M_{V}$ for the $\mathrm{M}$ dwarfs in our sample. We derived the $\left(M_{V}, V-R_{\mathrm{C}}\right)$ relation as follows: We first collected all of the stars with parallax data and $0.9<V-R_{\mathrm{C}}<1.65$ (no redder stars are contained in our sample) from the work of Leggett (1992). We then cross-referenced these stars with the Hipparcos Catalogue (ESA 1997) and substituted the Hipparcos parallax measurements when they were more precise. As a final cut, we removed unresolved, known double systems and stars with uncertainties in $M_{V}$ greater than $0.2 \mathrm{mag}$. Our resulting sample of 177 stars, along with our fit, is shown in Figure 2. The best-fit color-magnitude relation is

$$
M_{V}=-5.50+20.20\left(V-R_{\mathrm{C}}\right)-4.48\left(V-R_{\mathrm{C}}\right)^{2} .
$$

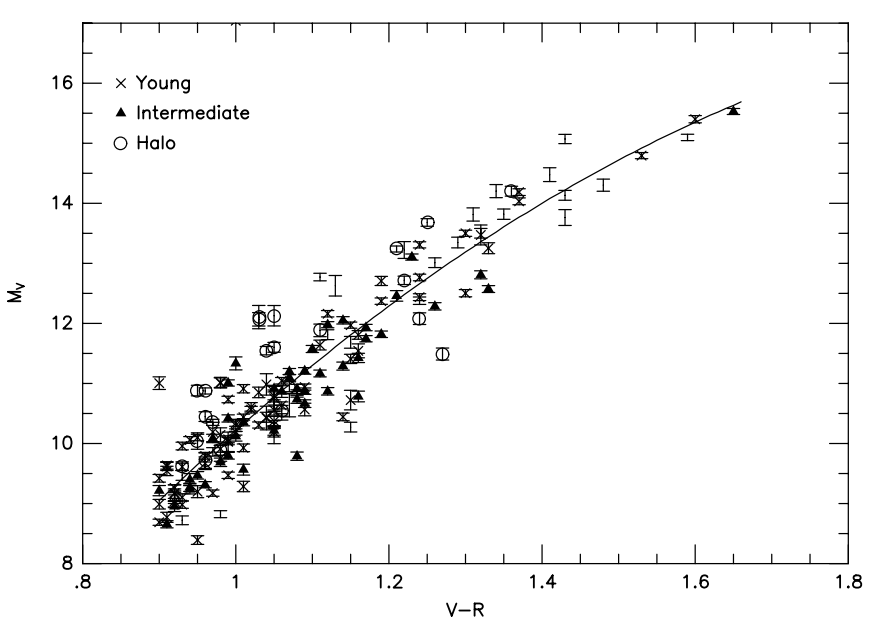

Fig. 2.-The color-absolute magnitude relation we derived for $\mathbf{M}$ dwarfs. This M dwarf sample is from the work of Leggett (1992), though we include new Hipparcos parallax data when available. $\mathbf{M}$ dwarfs that are kinematically members of the halo, intermediate, and young disk population are plotted as open circles, filled triangles, and crosses, respectively. We plot only error bars if the kinematic population type is not available. This relation is discussed in detail in $\S 3$. 
The unbiased mean deviation for the fit is $\sigma_{M_{V}} \sim 0.52$. We differentiate between members of the young (crosses), intermediate (triangles), and halo (circles) populations based on the kinematic data provided by Leggett (1992). We plot only error bars if the kinematic population membership was not available. We represent different kinematic populations with different symbols, as kinematic population type is known to correlate with metallicity (see, e.g., Carney, Latham, \& Laird 1990; Leggett 1992), which in turn may affect the color-magnitude relation because of the impact of the TiO bands on the $V$ and $R_{\mathrm{C}}$ bands. In our analysis, we find evidence for a different color-magnitude relation only in the halo population, which comprises approximately $12 \%$ of the calibration sample. We find that the halo stars are approximately $0.5 \mathrm{mag}$ fainter for a given $V-R_{\mathrm{C}}$ color. As metal-poor stars are expected not only to be fainter for a given $V-R_{\mathrm{C}}$, but may also contribute a relatively larger fraction of the stars observed farther from the Galactic plane, they may introduce a systematic effect. To see if our data show evidence for a metallicity gradient in the $M$ dwarf population, we examined the distribution of $B-V$ color at a given $V-R_{\mathrm{C}}$ color as a function of height above the plane. This analysis did not reveal a significant color change with height above the plane, and we therefore conclude from this result, and the relative scarcity of low-metallicity stars, that our results will not be strongly affected by a vertical metallicity gradient in the Galactic disk or a lowmetallicity component. This result is also consistent with the work of Reid et al. (1997), who concluded that there is not a significant metallicity gradient in the $\mathrm{M}$ dwarf population within $2 \mathrm{kpc}$ of the Galactic plane.

We note that most investigators (e.g., Stobie et al. 1989; Reid 1991; Gould et al. 1996) employ a linear relation between $V-I$ and $M_{V}$ to estimate stellar luminosities. Reid \& Gizis (1997) have shown, however, that there is a change in the slope of this relation at $V-I \sim 2.9$. They attribute much of the current disagreement on the shape of the stellar mass function to the use of an inexact $\left(M_{V}, V-I\right)$ relation.

\section{STELLAR DENSITY DISTRIBUTION}

The next step in our analysis was to use the calculated $M_{V}$ and the method of photometric parallax to estimate the perpendicular distance of our sample objects from the Galactic plane and their distance from the Galactic center. Stobie et al. (1989), in a study of the stellar luminosity function within $125 \mathrm{pc}$ and in the direction of the north Galactic pole, employed a single exponential distribution with a scale height of 325 pc (Yoshii, Ishida, \& Stobie 1987) to estimate the total effective volume (which they refer to as the generalized volume) sampled by their survey for the computation of the stellar luminosity function. Gould et al. $(1996,1997)$ have used the high angular resolution of $H S T$ to discriminate $\mathbf{M}$ dwarfs from galaxies many scale heights above the plane. Gould et al. $(1996,1997)$ used the method of maximum likelihood to model the vertical and radial distribution of the $\mathbf{M}$ dwarfs in their sample and found the vertical stellar density to be best fitted by a two-component model with a sech ${ }^{2}$ term and an exponential term, along with a parameter $\alpha$ to describe the relative contribution of each of these terms. Gould et al. also fitted a double exponential model to their data, but they found this did not provide as good agreement with their data as the $\operatorname{sech}^{2}+$ exponential model.
TABLE 2

Stellar Density Parameters

\begin{tabular}{ccc}
\hline \hline Parameter & Value & $\sigma$ \\
\hline$\alpha \ldots \ldots \ldots \ldots \ldots \ldots$. & 0.79 & 0.12 \\
$h_{1} \ldots \ldots \ldots \ldots \ldots \ldots$ & 340 & 40 \\
$h_{2} \ldots \ldots \ldots \ldots \ldots \ldots$ & 550 & 180 \\
\hline
\end{tabular}

Notes.-The parameters for our best-fit, twocomponent model of the vertical stellar density distribution. This model contains both a sech ${ }^{2}$ and an exponential term. The parameter $\alpha$ is the relative weight of the $\operatorname{sech}^{2}$ term; the exponential term is weighted by $1-\alpha$. Parameters $h_{1}$ and $h_{2}$ are the scale heights for the sech ${ }^{2}$ and the exponential distributions, respectively. The associated uncertainties, $\sigma$, correspond to the change in a given parameter that would raise the reduced $\chi^{2}$ by 1 with the other parameters held fixed. See $\S 4$ for more details.

Our sample includes a large number of stars within a kiloparsec of the Galactic plane. As we have a significantly larger number of stars in this range than does the HST sample of Gould et al. $(1996,1997)$, we have reexamined the functional form of the vertical stellar density distribution. Our modeling procedure was as follows: First, we selected all of the stars in our four brightest luminosity bins, $M_{V}=$ $9,10,11$, and 12 . We then separated out all stars within a kiloparsec of the Galactic plane and split these stars into $100 \mathrm{pc}$ bins. This set of four luminosity bins and 10 spatial bins was compared with the number of stars predicted by different models with a $\chi^{2}$ goodness-of-fit estimator. We used the downhill simplex method of Nelder \& Mead (1965) as described by Press et al. (1992) to test three models for the vertical stellar density distribution: a single exponential, a double exponential, and a sech ${ }^{2}+$ exponential model. We described the radial component in these models with the parameterization of Gould et al. (1997). Our search of the resulting five- or seven-dimensional parameter space found that the two-component $\operatorname{sech}^{2}+$ exponential model provided the best fit to our data. We list the best-fit parameters of this model in Table 2. Including stars farther above the plane or adding more luminosity bins to the model did not significantly change the best-fit parameters. Our resulting expression for the vertical stellar density distribution is

$$
v(z)=0.79 \operatorname{sech}^{2}\left(\frac{z}{340}\right)+0.21 \exp \left(\frac{-|z|}{550}\right) .
$$

We note that the parameters we derived for this model agree with the parameters derived by Gould et al. (1997) to within our uncertainties. After obtaining the above solution for the vertical distribution of stars, we attempted to solve for the radial distribution with a single-exponential fit of variable scale length. Our data were not, however, able to provide reasonable constraints on the disk scale length. We have therefore used the parameterization of the radial stellar density distribution derived by Gould et al. (1997):

$$
f(r)=\exp \left(\frac{8000-r}{2920}\right) \text {. }
$$

\section{ANALYSIS}

To calculate the stellar luminosity function, we used the concept of an effective volume to compute the total volume 
TABLE 3

\begin{tabular}{|c|c|c|c|}
\hline$V$ Limit & $V / V_{\max }$ & $\sigma_{V / V_{\max }}$ & $N$ \\
\hline $19.0 \ldots$ & 0.4963 & 0.0201 & 207 \\
\hline $19.5 \ldots$ & 0.4660 & 0.0170 & 291 \\
\hline $20.0 \ldots$ & 0.4727 & 0.0142 & 416 \\
\hline $20.5 \ldots$ & 0.5025 & 0.0116 & 618 \\
\hline $21.0 \ldots$ & 0.5136 & 0.0098 & 876 \\
\hline $21.5 \ldots$ & 0.5161 & 0.0084 & 1182 \\
\hline $22.0 \ldots$ & 0.5263 & 0.0073 & 1575 \\
\hline $22.5 \ldots \ldots$ & 0.5582 & 0.0062 & 2159 \\
\hline $23.0 \ldots \ldots \ldots \ldots$ & 0.5879 & 0.0053 & 2927 \\
\hline $23.5 \ldots$. & 0.6227 & 0.0046 & 4005 \\
\hline
\end{tabular}

NoTES.-A summary of the $V / V_{\max }$ calculations described in $\S 5$. Col. (1) lists the limiting $V$ magnitude of each sample. Cols. (2) and (3) report the value of $V / V_{\max }$ and the $1 \sigma$ uncertainties. Col. (4) contains the number of stars included in the sample.

within which a star of a given $M_{V}$ could have been detected. The effective volume is defined as

$$
v_{\text {eff }}\left(M_{V}\right)=\Omega \int_{d_{\min }}^{d} d l l^{2} v(z) f(r),
$$

where $\Omega$ is the angular size of the field in steradians, $v(z)$ and $f(r)$ are as defined above, and $l$ is the line element over which the star could have been detected from $d_{\text {min }}$, defined by the saturation limit in $V$, to $d$, derived from the distance modulus.

In order to refine the limiting magnitude of the survey, we used the fact that the stellar distribution should be uniform in effective volume space. We therefore computed the effective volume for each object from the saturation limit to its distance and divided this quantity by the effective volume within which the object could have been detected for a given limiting magnitude in $V$. If the survey is uncontaminated by small red galaxies down to a given limiting magnitude, the cumulative distribution of $v_{\text {eff }} / \max \left(v_{\text {eff }}\right)$ should be linear. If the survey suffers from an excess of objects near the limiting magnitude, then the cumulative distribution will appear to have positive curvature. Similarly, if the distribution is incomplete, then the cumulative distribution will appear to have negative curvature. Finally, a cumulative distribution that appears best fitted by a high-order polynomial would suggest that the model for the stellar distribution is incorrect. We computed the cumulative distribution for magnitude limits from $V=21$ to $V=24$. From this analysis, we conclude that there is insignificant contamination for $V \leq 22$ while, for $V>22$, the contamination of the stellar catalog quickly becomes significant.

A second test to determine the limiting $V$ magnitude at which the contamination of the $\mathrm{M}$ dwarf catalog begins to become severe is to use the $V / V_{\max }$ test of Schmidt (1968), as modified by Avni \& Bahcall (1980). The $V / V_{\max }$ test was applied by Reid et al. (1995) on the true space volume to estimate the completeness limit of a relatively nearby sample of $\mathbf{M}$ dwarfs from a preliminary version of the Gliese \& Jahreiss (1991) Third Catalogue of Nearby Stars as a function of $M_{V}$. Because of the density gradients in the stellar distribution, we calculated $V / V_{\max }$ with the effective volume, rather than the true space volume. Computing $V / V_{\max }$ for different limiting magnitudes is equivalent to testing for contamination or incompleteness at the faint end. If $V / V_{\max }$ is greater than 0.5 , this suggests the survey suffers from contamination of nonstellar objects at the faint end. Similarly, values less than 0.5 suggest incompleteness at the faint end. This method, however, is not as sensitive to errors in the assumed stellar distribution. Table 3 shows the results of the $V / V_{\max }$ calculations, where $V / V_{\max }$ is consistent with 0.5 within the $3 \sigma$ statistical fluctuations to approximately $V=22$.

\section{RESULTS}

The luminosity function, $\Phi\left(M_{V}\right)$, for a given absolute magnitude bin is

$$
\Phi\left(M_{V}\right)=\sum_{i=1}^{N} \frac{1}{\max \left[v_{\mathrm{eff}, i}\left(M_{V}\right)\right]},
$$

where $N$ is the number of stars in bin $M_{V}$. The resulting luminosity function is shown in Figure 3 for unit magnitude bins centered at integer magnitudes (open circles). We list the numerical values of both the luminosity and mass function in Table 4.

The bright end $\left(M_{V}<12\right)$ of the luminosity function is in excellent agreement with other recent measurements of the M dwarf luminosity function (Stobie et al. 1989; Reid \& Gizis 1997; Gould et al. 1997). The faint end of the luminosity function, however, has been significantly more controversial because of the selection effects and small number statistics, which complicate an accurate census of the faintest M dwarfs. Stobie et al. (1989) measured the luminosity function down to $M_{V}$ of 16 in a large-area $\left(21.46 \mathrm{deg}^{2}\right)$ survey of the north Galactic pole and found that the luminosity function peaks at $M_{V}=12$ and decreases over the range $12<M_{V}<15$, even when the effects of binary/ multiple systems are taken into account. Reid \& Gizis (1997) found a similar turndown in the their rederivation [using their new $\left(M_{V}, V-I\right)$ relation] of the luminosity

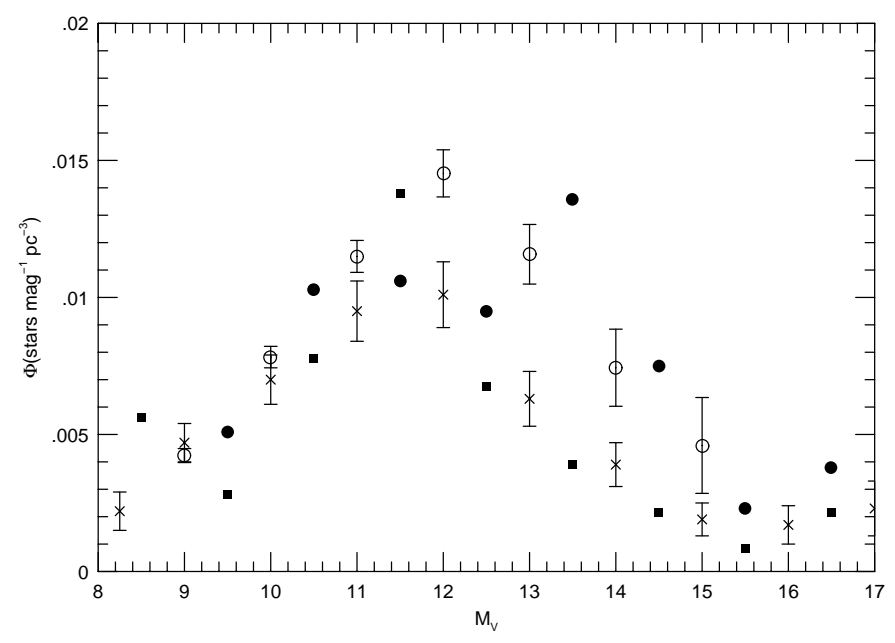

FIG. 3.-Stellar luminosity function of $M$ dwarfs at the Galactic plane as listed in Table 4. Open circles represent our derived stellar luminosity function; error bars are $1 \sigma$ uncertainties based on Poisson statistics. For comparison, we also plot the stellar luminosity function derived by Stobie, Ishida, \& Peacock (1989, filled squares), the rederived photometric parallax luminosity function of Reid \& Gizis (1997, filled circles), and the luminosity function of Gould, Bahcall, \& Flynn (1997, crosses with error bars). The data from Gould et al. (1997) are from their derivation based on naive binning. 
TABLE 4

Stellar Luminosity and Mass Functions

\begin{tabular}{crrcc}
\hline \hline$M_{V}$ & \multicolumn{1}{c}{$N$} & \multicolumn{1}{c}{$\Phi$} & $\log \left(M / M_{\odot}\right)$ & \multicolumn{1}{c}{ SMF } \\
\hline $9 \ldots \ldots \ldots \ldots \ldots$. & 292 & 4.2 & -0.239 & -1.06 \\
$10 \ldots \ldots \ldots \ldots \ldots$. & 415 & 7.8 & -0.289 & -0.98 \\
$11 \ldots \ldots \ldots \ldots \ldots$. & 397 & 11.5 & -0.427 & -1.16 \\
$12 \ldots \ldots \ldots \ldots \ldots$. & 299 & 14.5 & -0.596 & -1.05 \\
$13 \ldots \ldots \ldots \ldots \ldots$. & 126 & 11.6 & -0.755 & -1.00 \\
$14 \ldots \ldots \ldots \ldots \ldots$. & 37 & 7.4 & -0.849 & -0.95 \\
$15 \ldots \ldots \ldots \ldots \ldots$ & 9 & 4.6 & -0.931 & -1.07 \\
\hline
\end{tabular}

Notes.-Stellar luminosity and mass function derived with this sample. The stellar density has been normalized to the local neighborhood. Cols. (1) and (2) list the center of each magnitude bin and the corresponding number of stars in this bin, while col. (3) lists the stellar luminosity function $\left(10^{-3}\right.$ stars $\left.\mathrm{pc}^{-3}\right)$. Col. (4) lists $\log \left(M / M_{\odot}\right)$ corresponding to the $M_{V}$ given in col. (1), while col. (5) contains the stellar mass function $\left\{\log \left[\operatorname{stars~pc^{-3}} \log \left(M / M_{\odot}\right)^{-1}\right]\right\}$.

function from photometric parallax for $M_{V}=12-15$, though with an increase again for the very faintest dwarfs. We note, however, that the increase in the photometric luminosity function for $M_{V}>15$ has a very low statistical weight. Gould et al. (1997) also find that the luminosity function peaks at $M_{V}=12$, though they also see some evidence for an increase in the stellar number density for the faintest $\left(M_{V}>16\right) M$ dwarfs. In agreement with these investigators, we find the $\mathbf{M}$ dwarf luminosity function turns over at $M_{V} \sim 12$ and declines to at least $M_{V}=15$, the limit of our sample. The number density we measure at the faint end, shown in Figure 3, is greater than that of Gould et al. (1997, crosses with error bars) and Stobie et al. (1989, filled triangles), and slightly less than that of Reid \& Gizis (1997, filled circles).

Including $\mathbf{M}$ dwarfs in binary systems introduces two competing effects. Unresolved, red binaries can artificially increase the luminosity function by scattering stars into the sample (Reid et al. 1995), leading to an underestimated distance and correspondingly overestimated space density. In contrast, unobserved $\mathbf{M}$ dwarf secondaries are missed, leading to an underestimation of their true space density. Reid \& Gizis (1997) used their measurement of the binary fraction within $8 \mathrm{pc}$ to model the effect of unresolved binaries on the luminosity function. They find that the luminosity function derived via the technique of photometric parallax is in general agreement with the true luminosity function used as input to their model.

To convert our luminosity function into a mass function, we adopted the empirical mass- $M_{V}$ relation of Henry \& McCarthy (1993). The resulting $M$ dwarf mass function is shown in Figure 4. As in Figure 3, we have plotted the bins centered on integer magnitudes (open circles). We find the mass function to be essentially flat from the highest mass $\mathbf{M}$ dwarfs to $M \sim 0.1 M_{\odot}$. Quantitatively, a power-law fit to our data over the range $0.6 M_{\odot}>M>0.1 M_{\odot}$ yields an index $\alpha=-0.32 \pm 0.15$, where $\alpha=d \log N / d \log M$.

A deviation from a Salpeter mass function for $M<0.6$ $M_{\odot}$ has been previously reported by Miller \& Scalo (1979), Kroupa, Tout, \& Gilmore (1993), Reid et al. (1995), and Gould et al. (1996). Gould et al. (1997) find the break in the power law to occur at $M=0.6 M_{\odot}$. At the high-mass end, they measure a power-law index $\alpha=-1.21$ based on the luminosity function of Wielen, Jahreiss, \& Krüger (1983). For $M<0.6 M_{\odot}$, they find $\alpha=0.44$ to provide the best fit

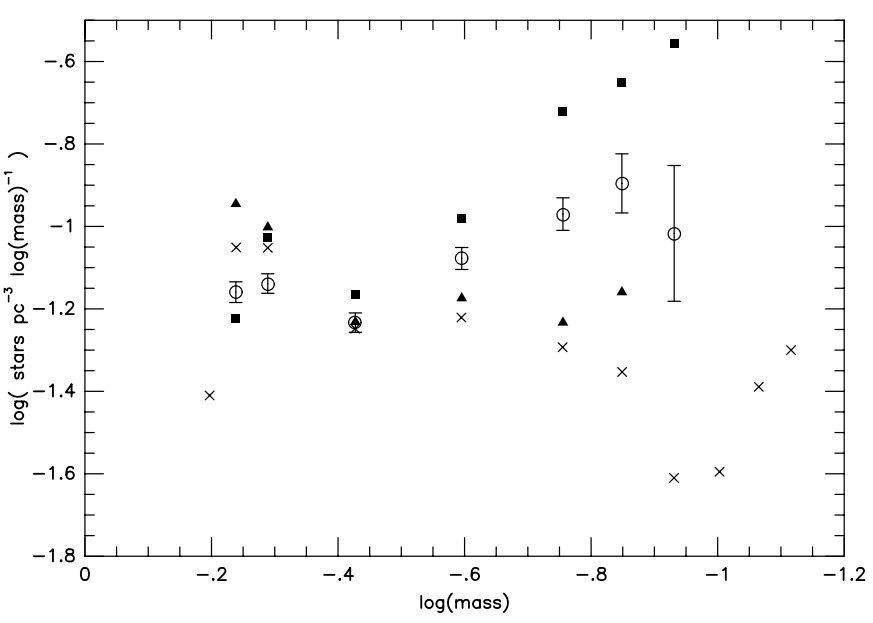

Fig. 4.- $\mathrm{M}$ dwarf mass function, derived by employing the massluminosity relation of Henry \& McCarthy (1993). As in Fig. 2, open circles represent our derived mass function and crosses represent the mass function derived by Gould et al. (1997). We also plot the change in our derived mass function if we vary our color-magnitude relation by $+1 \sigma$ (filled squares) or $-1 \sigma$ (filled triangles); see text.

to their HST sample. Reid \& Gizis (1997) find the mass function to be flat $(\alpha=-0.05)$ over the mass range 1-0.1 $M_{\odot}$ for stars within $8 \mathrm{pc}$.

As stated above, a change in our adopted colormagnitude relation can affect the shape of the luminosity function; similarly, any uncertainties in this relation could alter the shape of the mass function. We explored the direction and magnitude of this effect by varying the colormagnitude relation by $1 \sigma$ and rederiving the mass function. We found these systematic changes to have a negligible effect on the mass function for $M>0.25 M_{\odot}$, while at the low-mass end they can significantly affect the steepness of the power-law slope.

Previous investigators have found evidence for an upturn in the mass function near the hydrogen burning limit. We do not find any stars with $M_{V} \geq 16$ and are unable to comment on the shape of the mass function in this regime. Extrapolating the luminosity function of Gould et al. (1997) to our sample leads us to expect on the order of one star beyond $M_{V} \sim 15$.

\section{CONCLUSIONS}

We have used a combination of multicolor photometry and morphological information to extend a search for $\mathbf{M}$ dwarfs to fainter apparent magnitudes than previously possible without the additional observing overhead of spectroscopy. This catalog includes $1575 \mathrm{M}$ dwarfs, including 499 in bins over the range $12 \leq M_{V} \leq 15$, and thus provides a good statistical sample of these faint stars near the hydrogen burning limit. Our derived luminosity and mass function for $\mathbf{M}$ dwarfs is consistent with previous work in that it finds a definite turnover in the stellar luminosity function fainter than $M_{V}=12$. For $M_{V}<12$ our results are in good agreement with other investigators'. For stars fainter than the turnover, our estimated space densities lie between the ground-based photometric parallax values of Reid \& Gizis (1997) and the HST results of Gould et al. (1997). Our mass function is relatively flat for $M<0.6 M_{\odot}$ and is inconsistent with a Salpeter function at the $3 \sigma$ level. We thus con- 
clude that the mass function significantly flattens out in the $\mathbf{M}$ dwarf mass regime, in broad agreement with the work of Reid \& Gizis (1997) and Gould et al. (1997).

The technique we have presented in this paper can easily be applied to other large-area, ground-based surveys. We have shown that multicolor photometry alone can be used to discriminate $\mathbf{M}$ dwarfs from galaxies to at least $V \sim 22$. Many square degrees could thus be efficiently surveyed with modern large-format cameras to collect a large sample of the faintest M dwarfs. Such a survey would provide the necessary statistics to improve the parameterization of the M dwarf density distribution and to accurately determine the shape of the mass function near the hydrogen burning limit.

We would like to thank Andy Gould for several helpful suggestions and comments on this manuscript. We would also like to thank the referee for several valuable suggestions that have improved this paper and our presentation. In addition, we would like to thank Pat Hall for providing additional information on his work with this survey. This research has made use of the SIMBAD database, operated at CDS, Strasbourg, France. This work was supported in part by NSF grant AST 95-29324 to P. S. O.
Avni, Y., \& Bahcall, J. N. 1980, ApJ, 235, 694

Bahcall, J. N., Flynn, C., Gould, A., \& Kirhakos, S. 1994, ApJ, 435, L51

Carney, B. W., Latham, D. W., \& Laird, J. B. 1990, AJ, 99, 572

ESA. 1997, The Hipparcos and Tycho Catalogues (ESA SP-1200) (Noordwijk: ESA)

Gliese, W., \& Jahreiss, H. 1991, Preliminary Version of the Third Catalogue of Nearby Stars (NSSDC/ADC Cat. 5070A) (Greenbelt, MD: GSFC)

Gould, A., Bahcall, J. N., \& Flynn, C. 1996, ApJ, 465, 759 .1997, ApJ, 482, 913

Hall, P. B., Osmer, P. S., Green, R. F., Porter, A. C., \& Warren, S. J. 1996a, ApJS, 104, 185

Henry, T. J., \& McCarthy, D. W., Jr. 1993, AJ, 106, 773

Kennefick, J. D., Osmer, P. S., Hall, P. B., \& Green, R. F. 1997, AJ, 114, 2269

Kirkpatrick, J. D., McGraw, J. T., Hess, T. R., Liebert, J., \& McCarthy, D. W., Jr. 1994, ApJS, 94, 749

Koo, D. C., Kron, R. G., \& Cudworth, K. M. 1986, PASP, 98, 285

Kroupa, P., Tout, C. A., \& Gilmore, G. 1993, MNRAS, 262, 545

Leggett, S. K. 1992, ApJS, 82, 351

\section{REFERENCES}

Liu, C. T., Green, R. F., Hall, P. B., \& Osmer, P. S. 1998, AJ, in press

Miller, G. E., \& Scalo, J. M. 1979, ApJS, 41, 513

Nelder, J. A., \& Mead, R. 1965, Comput. J., 7, 308

Osmer, P. S., Kennefick, J. D., Hall, P. B., \& Green, R. F. 1998, ApJS, in press

Press, W. H., Teukolsky, S. A., Vetterling, W. T., \& Flannery, B. P. 1992, Numerical Recipes in C (2d ed.; Cambridge: Cambridge Univ. Press)

Reid, I. N., \& Gizis, J. E. 1997, AJ, 113, 2246

Reid, I. N., Gizis, J. E., Cohen, J. G., Pahre, M. A., Hogg, D. W., Cowie, L., Hu, E., \& Songaila, A. 1997, PASP, 109, 559

Reid, I. N., Hawley, S. L., \& Gizis, J. E. 1995, AJ, 110, 1838

Reid, I. N., Yan, L., Majewski, S., Thompson, I., \& Smail, I. 1996, AJ, 112, 1472

Reid, N. 1991, AJ, 102, 1428

Schmidt, M. 1968, ApJ, 151, 393

Stobie, R. S., Ishida, K., \& Peacock, J. A. 1989, MNRAS, 238, 709

Wielen, R., Jahreiss, H., \& Krüger, R. 1983, in IAU Colloq. 76, The Nearby Stars and the Stellar Luminosity Function, ed. A. G. D. Philip \& A. R. Upgren (Schenectady, NY: L. Davis), 163

Yoshii, Y., Ishida, K., \& Stobie, R. S. 1987, AJ, 93, 323 